\title{
Low-Voltage Operation Photodetector Made by Coupling Superlattice and Quantum Wells
}

\author{
J. H. Lu ${ }^{1}$, Y. C. Wang, C. L. Wang, C. H. Kuan ${ }^{2}$ \\ Graduate Institute of Electronics Engineering and Department of electrical engineering, National Taiwan University \\ No.1, Sec. 4, Roosevelt Road, Taipei, Taiwan, Republic of China 10617 \\ ${ }^{1}$ d91943025@ntu.edu.tw ${ }^{2}$ kuan(a)cc.ee.nu.edu.tw \\ C. W. Yang, S. L. Tu \\ Opto Tech Corporation, I, Li-hsin Rd. V. Hsinchu Science-based industrial Park, Hsinchu, Taiwan, Republic of China
}

\begin{abstract}
We have investigated a novel photodetector structure of coupling superlattice and quantum wells. This device can be operated at low bias range and even the photovoltaic mode. The broadband response is achieved by this structure.

(C) 2005 Optical Society of America

OCIS codes: $(040.3060)$ Infrared ; (230.5590) Quantum-well devices
\end{abstract}

\section{Introduction}

In recent years, the applications such as the focal plane array (FPA) and remote temperature sensing drive the research of novel intersubband transition devices. Some useful characteristics of quantum well infrared photodetector (QWIP) including multicolor, photovoltaic are also investigated [1-3]. In addition to QWIP, the superlattice infrared photodetector (SLIP) is another promising structure to achieve those applications. Compared with the quantum well, superlattice has the properties including low power consumption, broadband photoresponse and voltage-tunable. The performance of SLIP has been proved by our group [4-6]. The perspective of our detector is to operate at low bias, therefore, low power consumption and low dark current are expected. We also hope the operation temperature can be higher and the broadband and flatband spectrum can be observed. Based on our perspective, a new structure of superlattice coupled to quantum wells is designed.

Figures 1(a) and (b) show the band diagrams of our sample under photovoltaic and photoconductive mode, respectively. Under zero bias, the photovoltaic mode, electrons in superlattice excited to the second miniband will go through the barrier by group velocity and captured by some quantum wells. Because of those captured electrons, the potential on superlattice side is relatively positive and the quantum well is negative. Therefore, a built-in potential will exist and the energy band will be bended as shown in Fig. 1(a). On the other hand, due to the dopant migration during the growth process, a built-in electric field is created at the barrier in quantum well structure. Then, electrons in quantum well excited to the bound state or the continuum band can tunnel through the barrier and become the photocurrent. Hence, under zero bias, the photocurrent from superlattice and quantum wells can be measured simultaneously.

For the photoconductive mode, Fig. 1(b) shows our sample operated under negative bias. When operated under negative bias, this structure works just as the general photodetector. Electrons in superlattice excited by infrared radiation will go through the barrier and be accelerated by the electrical field on the quantum well structure. At the same time, electrons in quantum well can also overcome the barrier and then become the photocurrent. On the other hand, under positive bias, electrons will be attracted to top contact. In the superlattice, the electrons transport to top contact and leave the superlattice a positive electric field. Therefore, electrons will be attracted back to superlattice and become an internal circulation which cannot be detected. So we can measure both the superlattice and quantum well response under negative bias, but under positive bias we can only observe the QWIP response.

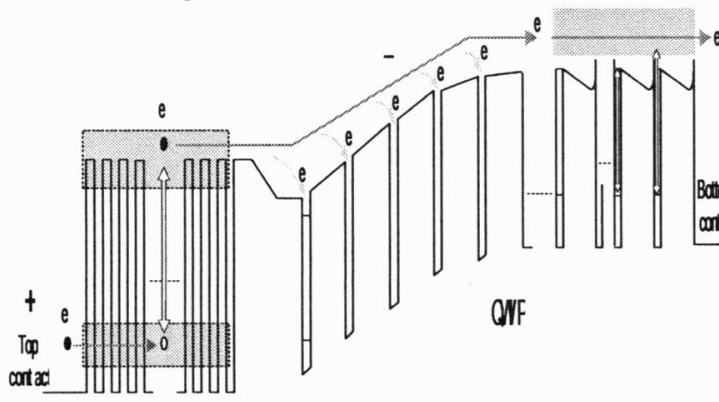

\/F

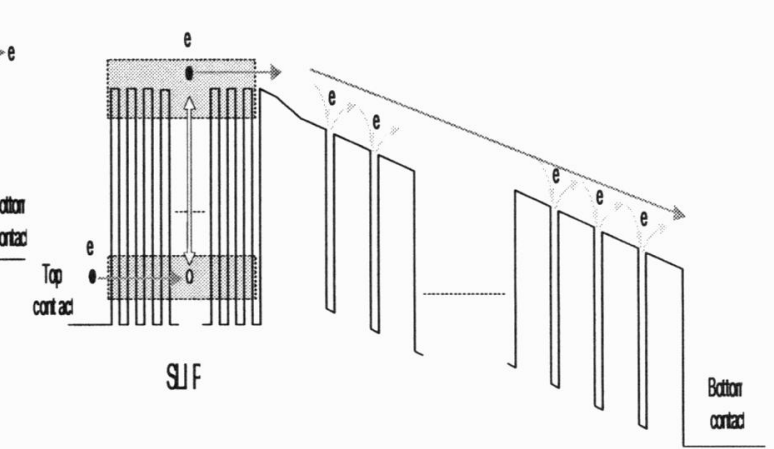

QWF

(a) (b)

Fig. 1. The band diagram under (a)photovoltaic (PV) mode and (b)photoconductive (PV) mode of negative bias.

\section{Sample Structure}




\section{JWB31}

Our sample structure contains a 15-period superlattice, a $60 \mathrm{~nm}$ graded barrier and a 50 -period quantum well. Each period of the quantum well consists of $6 \mathrm{~nm}$ GaAs well and $50 \mathrm{~nm}$ undoped $\mathrm{Al}_{0.21} \mathrm{Ga}_{0.79} \mathrm{As}$ barrier and each period of superlattice consists of $6 \mathrm{~nm}$ GaAs well and $4 \mathrm{~nm}$ undoped $\mathrm{Al}_{0.29} \mathrm{Ga}_{0.71}$ As barrier. The superlattice and quantum well structure are both doped with $\mathrm{Si}$ for $4 \times 10^{17} \mathrm{~cm}^{-3}$. The absorption wavelength of superlattice we designed is $6 \sim 10 \mu \mathrm{m}$, and the transition type for quantum well is bound to continuum and the longest absorption wavelength is $11.7 \mu \mathrm{m}$.

\section{Experimental Results and Discussions}

Figures 2(a) and (b) are the photoresponse under photovoltaic mode at low temperature and high temperature, respectively. The cause of the photovoltaic response from superlattice and quantum well are group velocity and the dopant migration, respectively. Because the photovoltaic spectrums are measured under open-circuit condition by voltage amplifier, the vertical axis in both figures is the relative voltage amplitude. Three peaks can be distinguished from the spectrum. One short wavelength peak is at $6.5 \mu \mathrm{m}$ from superlattice and the other two attributed to the quantum wells are located at $9.2 \mu \mathrm{m}$ and $11.5 \mu \mathrm{m}$. As temperature rising, the superlattice response is steady enhanced, while the quantum well response is gradually diminished. From Fig. 2(b), the superlattice response reaches the maximum at $60 \mathrm{~K}$. At high temperature, due to the phonon scattering increases, the relaxation process in quantum wells becomes more serious, so the quantum well response is almost disappeared. The arrows in both figures show the trend of the superlattice response.

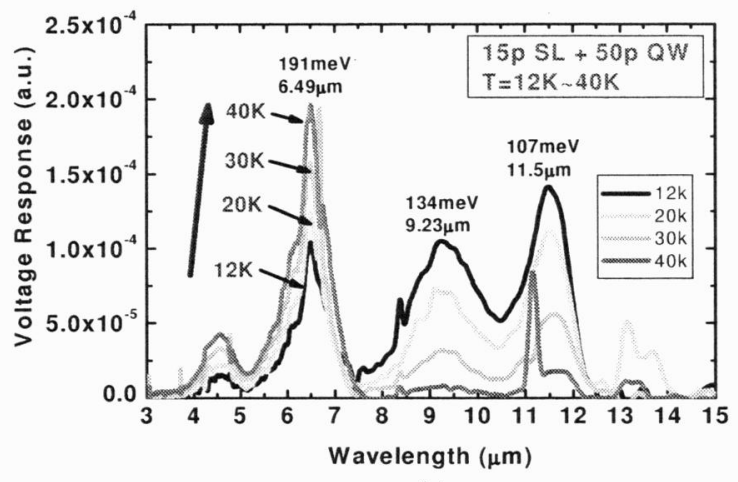

(a)

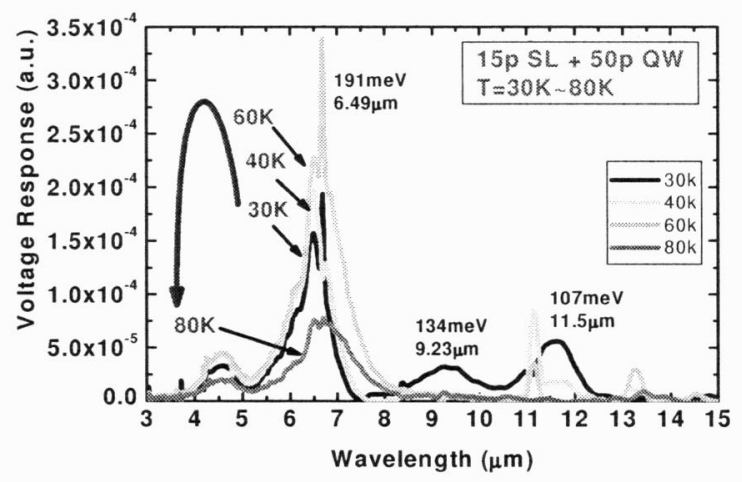

(b)

Fig. 2. The photovoltaic response under (a)low and (b)high temperature. The arrow shows the trend of the superlattice response as temperature changing. The response from QWIP is gradually reduced as temperature rising.

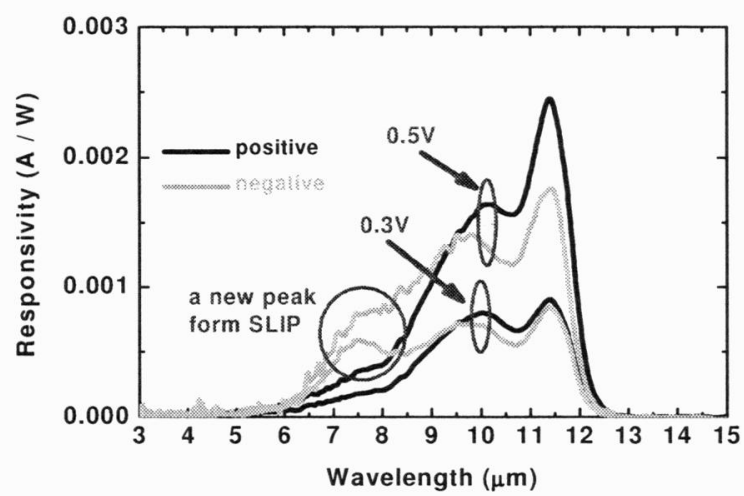

(a)

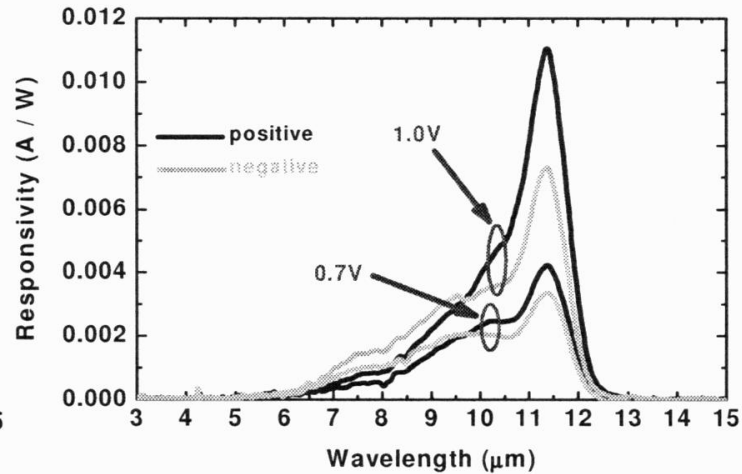

(b)

Fig. 3. The comparison of responses at $12 \mathrm{~K}$ under negative bias to those under positive bias at (a) $0.3 \mathrm{~V}$ and $0.5 \mathrm{~V}$, (b) $0.7 \mathrm{~V}$ and $1.0 \mathrm{~V}$. The short wavelength peak from SLIP can be clearly distinguished under negative bias, hence the response under negative bias is more broadband than the positive one.

Figures 3(a) and (b) are the comparison of the responses under negative bias to those under positive bias at different bias magnitude. From Fig. 3(a), the photoresponse under positive bias from only quantum wells can be divided into two parts. The first part is the response at short wavelength side including a peak at $10.1 \mu \mathrm{m}$ and a tail at shorter wavelength. The other part is the peak at $11.4 \mu \mathrm{m}$. Because the transition type of quantum wells is bound-to-continuum, we consider the short wavelength part is from the bound-to-continuum and the $11.4 \mu \mathrm{m}$ peak is from bound-to-bound transition. For bias higher than 1.0V, as Fig. 3(b) shown, only the bound-to-bound peak can be observed.

For a bound-to-continuum QWIP, a broadband detector can also be achieved. However, the intensity of the short wavelength tail is relatively weak. Therefore, our perspective of broadband and flatband response can be 
achieved by adding the SLIP designed for short wavelength detection into the structure.

Under negative bias, the spectrum is the combination of the response from superlattice and quantum wells, and a new peak at short wavelength side from superlattice can be clearly distinguished when compared to spectrum under positive bias. For the response under $0.3 \mathrm{~V}$, the response under negative bias is more broadband than that under positive bias. The additional spectrum located around $7 \mu \mathrm{m}$ is from superlattice as our design and the other two peaks from quantum wells are just like the response under positive bias. As bias increases, the broadband response under negative bias can still be observed but less apparent. Since most of the sample structure is quantum wells, at high negative bias, only one peak from bound-to-bound transition in quantum wells exists like the response under positive bias. The above described photovoltaic and voltage-tunable characteristics can be observed until $80 \mathrm{~K}$.

The detectivity of our sample at three different temperature is shown in Fig.4. Here we choose two different wavelengths for the detectivity under positive and negative bias. For positive bias, the origin of response is quantum wells, so detectivity at $11.4 \mu \mathrm{m}$ is shown. For low negative bias range, the response is mainly from the superlattice. Hence, the wavelength of the negative bias detectivity is chosen as $7.4 \mu \mathrm{m}$. From Fig.4, we observe the maximum detectivity occurred at low negative bias. At different temperature, the peak value of negative bias detectivity from superlattice is comparable to the detectivity from quantum wells even the bias at $2 \mathrm{~V}$. It is the evidence the superlattice suits for the low bias operation. Besides, this phenomenon is observed even at high temperature as $90 \mathrm{~K}$. Therefore, the superlattice is also ideal for high temperature operation.

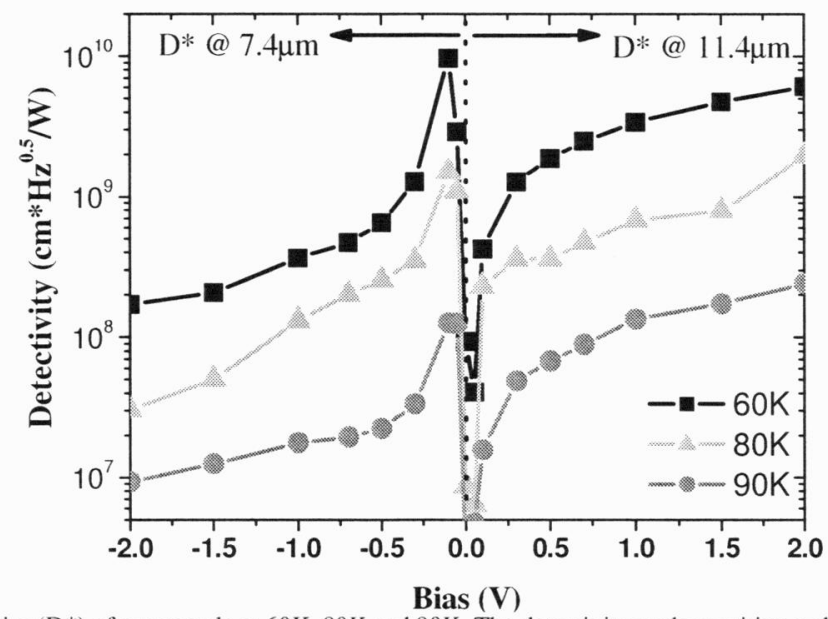

Fig. 4. The detectivity $\left(D^{*}\right)$ of our sample at $60 \mathrm{~K}, 80 \mathrm{~K}$ and $90 \mathrm{~K}$. The detectivity under positive and negative bias are at 11.4 and $7.4 \mu \mathrm{m}$, respectively.

\section{Conclusion}

In conclusion, a new structure of SLIP coupled to QWIP is investigated. By using this structure, the photovoltaic operation mode is achieved because the dopant migration effect in quantum wells and group velocity in superlattice. For photoconductive mode, only QWIP response exists at positive bias. Under low negative bias, the photoresponse from both SLIP and QWIP can be observed. By using this structure, under low bias range, the detector is not only with low dark current for lower power consumption but also the broadband and flatband photoresponse. This structure also makes the high temperature operation possible. Therefore, another choice is provided to implement the focal plane array.

\section{References}

[1] L. C. Lenchyshyn, H. C. Liu, M. Bunchanan and Z. R. Wasilewski, "Voltage-tuning in multi-color quantum well infrared photodetector stacks," J. Appl. Phys. 79, 8091 (1996)

[2] K. L. Tsai, K. H. Chang, C. P. Lee, K. F. Huang, J. S. Tsang and H. R. Chen, "Two-color infrared photodetector using GaAs/AlGaAs and strained InGaAs/AlGaAs multiquantum wells," Appl. Phys. Lett. 62, 3504-3506 (1993)

[3] Yaohui Zhang, D. S. Jiang, J. B. Xia, L. Q. Cui, C. Y. Song, Z. Q. Zhou and W. K. Ge, “A voltage-controlled tunable two-color infrared photodetector using GaAs/AlAs/GaAlAs and GaAs/GaAlAs stacked multiquantum wells," Appl. Phys. Lett. 68, 2114-2116 (1996)

[4] C. C. Chen, H. C. Chen, M. C. Hsu, W. H. Hsieh, C. H. Kuan, S. Y. Wang and C. P. Lee, "Performance and application of a superlattice infrared photodetector with a blocking barrier," J. Appl. Phys. 91, 943-948 (2003)

[5] C. C. Chen, H. C. Chen, C. H. Kuan, S. D. Lin and C. P. Lee, "Multicolor infrared detection realized with two distinct superlattices separated by a blocking barrier," Appl. Phys. Lett. 80, 2252-2254 (2002)

[6] J. H. Lu, Y. Y. Yang, C. C. Chen, C. H. Kuan, H. T. Chen and S. C. Lee, "Study of period number effect in the superlattice infrared photodetector," Infra. Phys. \& Technol. 44, 399-409 (2003) 